\title{
DETERMINAÇÃO DE METAIS POR TÉCNICAS DE ESPECTROMETRIA ATÔMICA EM AMOSTRAS DE CARVÃO MINERAL E CINZAS UTILIZANDO METODOLOGIA DE SUPERFÍCIE DE RESPOSTA
}

\author{
Camila M. Oliz, Emanoelli R. Lopes, Aline L. Medina, Anderson S. Ribeiro e Adriane M. Nunes* \\ Centro de Ciências Químicas, Farmacêuticas e de Alimentos, Universidade Federal de Pelotas, Campus Capão do Leão, 96160-000 \\ Capão do Leão - RS, Brasil
}

Recebido em 21/12/2015; aceito em 10/05/2016; publicado na web em 13/07/2016

\begin{abstract}
METALS DETERMINATION BY ATOMIC SPECTROMETRY TECHNIQUES IN COAL AND ASH SAMPLES USING RESPONSE SURFACE METHODOLOGY. This paper describes the method optimization using response surface methodology for $\mathrm{Cu}, \mathrm{Fe}, \mathrm{Zn}$ and $\mathrm{Na}$ determinations in coal and ash samples by spectrometry techniques. The sample preparation was performed using a recirculation system adapted in the digestion tubes. This system allowed increase of temperature in the digester block above the boiling point of the reaction medium, avoiding acid evaporation during the heating. The digestions were realized with $\mathrm{HNO}_{3}$ and $\mathrm{H}_{2} \mathrm{O}_{2}$ for $1 \mathrm{~h}$ in a digester block at $145^{\circ} \mathrm{C}$. The limits of detection for determination of $\mathrm{Cu}, \mathrm{Fe}, \mathrm{Zn}$ and $\mathrm{Na}$ in both samples were satisfactory. The RSD for the analytes were lower than $6.0 \%$. Statistical tests showed that results for all analytes do not vary significantly with official method (ASTM), considering a confidence limit of $95 \%$. Besides, the accuracy was verified by recovery tests, whose values ranged from 92.7 to $104.7 \%$ in coal, and 98.7 to $115.3 \%$ in ash.
\end{abstract}

Keywords: coal; ash; metals; atomic spectrometry; validation.

\section{INTRODUÇÃO}

O carvão mineral está entre os principais combustíveis fósseis utilizados mundialmente, ocupando a primeira colocação em abundância, o que representa $67 \%$ das reservas dos recursos energéticos do Brasil. Na composição da matriz energética global, o carvão fica abaixo apenas do petróleo, sendo que especificamente na geração de eletricidade passa à condição de principal recurso mundial. ${ }^{1}$ Deste modo, a produção de energia térmica tem se apresentado como uma alternativa técnica e economicamente viável ao sistema elétrico nacional, tendo em vista que os recursos hídricos disponíveis estão cada vez mais escassos e outros meios competitivos ainda não são suficientes para suprir esta demanda. ${ }^{2}$

No entanto, para a geração de energia elétrica através deste combustível fóssil são necessárias grandes quantidades, o que resulta na produção de elevadas porções de resíduos, como as cinzas. Consequentemente, muitos problemas ambientais podem ser atribuídos à combustão do carvão, principalmente os que estão relacionados à mobilização de constituintes orgânicos e inorgânicos potencialmente tóxicos decorrentes destes subprodutos, podendo causar sérios impactos ambientais, resultando na contaminação do solo, da água e do ar. $^{3}$

O carvão é constituído por uma mistura complexa de alto teor orgânico, associado a componentes inorgânicos (minerais), como os silicatos, sulfetos, carbonatos, óxidos, entre outros. ${ }^{4}$ Esta matriz associa vários elementos traço em sua composição e o conhecimento deste conteúdo pode fornecer informações para avaliar o risco ambiental embutido. ${ }^{5,6}$ As propriedades das cinzas estão diretamente relacionadas às características dos carvões que lhe dão origem, tornando de suma relevância a determinação de metais. Tendo em vista que as cinzas são consideradas resíduos e sua devida classificação se faz necessária, os limites máximos de alguns metais como $\mathrm{Al}, \mathrm{Ba}, \mathrm{Cu}$, $\mathrm{Fe}, \mathrm{Mn}, \mathrm{Na}, \mathrm{Zn}$, devem atender a normativa vigente. ${ }^{7}$

A transformação de uma amostra sólida em uma solução, já que a maioria das técnicas analíticas requer a dissolução completa, pode

*e-mail: adriane.mn@hotmail.com ser realizada por diferentes formas de decomposição, sendo que os processos mais conhecidos são a decomposição por via úmida, por via seca e por fusão. ${ }^{89}$ Além dessas possibilidades na decomposição de amostras sólidas (como carvão e cinzas), existem alternativas que também apresentam bons resultados, entre elas estão as extrações ácidas assistidas por ultrassom ou micro-ondas, ${ }^{10,11}$ obtenção de suspensões, ${ }^{12-15}$ ou determinação direta em amostragem sólida. ${ }^{16,17}$ Porém, na maioria dos casos, com o uso das suspensões e amostragem sólida se faz necessário uma instrumentação apropriada para a introdução da amostra, com detectores e sistemas de correção de fundo de alta resolução, a fim de corrigir possíveis erros provocados por interferências associados à presença da matriz da amostra durante a análise.

De acordo com a American Society for Testing e Materials (ASTM), para a caracterização das amostras de carvão por técnicas de espectrometria atômica recomenda-se inicialmente uma etapa de calcinação seguida por digestão das cinzas resultantes com uma mistura de água régia e posterior dissolução em ácido nítrico ou o ácido fluorídrico. Em alguns casos, a amostra também pode ser dissolvida com ácido nítrico ou com uma mistura de ácido clorídrico/fluorídrico seguido do uso do ácido bórico. ${ }^{18-20}$

A decomposição assistida por micro-ondas em sistemas fechados tem sido amplamente utilizada para a mineralização de amostras de carvão. Isto se deve pelo o método apresentar alta eficiência de decomposição para diferentes tipos de amostras, aliada com o baixo consumo de reagentes, bem como a redução de riscos de perdas de analitos e contaminação em comparação com procedimentos de digestão convencionais..$^{21-23}$ Entretanto, estes instrumentos ainda são de difícil acesso para muitos laboratórios, o que está associado ao seu alto valor aquisitivo. Além disso, por questão de segurança, apresentam a limitação do uso de pequenas massas de amostras, o que em alguns casos é um fator limitante para muitas análises.

Recentemente vem se utilizando para a decomposição ácida de diversas matrizes o uso de um sistema de refluxo (com dedo frio). ${ }^{24-}$ ${ }^{26}$ Esse sistema apresenta-se como uma alternativa promissora na substituição dos métodos convencionais, já que reduz quantidade de reagentes e evita o risco de contaminação e perdas por volatilização, além de possuir menor custo. 
A metodologia de planejamento fatorial associada à analise de superfícies de respostas é uma ferramenta fundamentada na teoria estatística, que fornece informações seguras sobre o processo, minimizando o empirismo que envolve técnicas de tentativa e erro. ${ }^{27}$ Como vantagens, avalia a influência das variáveis sobre as respostas desejadas, permitindo a otimização de processos e metodologias analíticas, que, através de uma condição otimizada, reduz custos e tempo, possibilita menor uso de reagentes e amostra, melhorando a eficiência dos processos. Diferentes metolodologias de planejamentos fatoriais podem ser utilizados, como Plackett \& Burman, Box Hunter, Doehlert, sendo que o DCCR (delineamento composto central rotacional) permite a avaliação das variáveis com um menor número de ensaios, além da construção de gráficos de superfícies de resposta.

Dentro deste contexto, fica evidente a necessidade do desenvolvimento e da validação de novas metodologias analíticas, alternativas aos métodos já existentes, para a determinação de metais em amostras de carvão e cinzas, focadas principalmente a etapa de preparo de amostras. Sendo assim, o objetivo deste trabalho foi desenvolver um método simples, rápido e de baixo custo de preparo das amostras de carvão mineral e cinzas, utilizando decomposição ácida em sistema de refluxo para a determinação de $\mathrm{Cu}, \mathrm{Fe}, \mathrm{Na}$ e $\mathrm{Zn}$ por técnicas de Espectrometria Atômica (F AAS e F AES). Para este estudo foi utilizado planejamento estatístico visando a otimização das variáveis relacionadas ao método proposto objetivando uma condição compromisso para os respectivos analitos.

\section{PARTE EXPERIMENTAL}

\section{Equipamentos e parâmetros instrumentais}

As determinações foram feitas em um Espectrômetro de Absorção Atômica com chama (Annalyst 200, Perkin Elmer). Foram utilizadas lâmpadas de catodo oco de $\mathrm{Cu}$, Fe e $\mathrm{Zn}$, operando nas condições fixadas pelo fabricante, nos comprimentos de onda: 324,$75 ; 248,33$; 213,86 nm; respectivamente, e uma lâmpada de arco de deutério como corretor de fundo. Para a determinação do Na foi empregado o modo emissão, sendo utilizado o comprimento de onda de $589 \mathrm{~nm}$. Ar e acetileno 99,7\% (Linde, São Paulo, Brasil) foram utilizados na chama.

As amostras foram trituradas em um gral de porcelana e peneiradas utilizando um agitador de peneiras AG-G1712 (Bronzinox, São Paulo, Brasil) e peneira de malha 270, $53 \mu \mathrm{m}$ (Bronzinox, São Paulo, Brasil). Após esta etapa, as amostras foram pesadas utilizando uma balança analítica com resolução de $0,1 \mathrm{mg}$ e tara máxima de $210 \mathrm{~g}$ (Modelo AR 2140, Ohaus Adventurer, EUA). Para o processo de decomposição ácida das amostras foi utilizado um bloco digestor convencional (modelo MA-4025, Marconi, Brasil). Em cada um dos tubos digestor foi introduzido um sistema de refluxo (Figura 1, cold finger) com circulação de água. $\mathrm{O}$ sistema consiste de um tubo de vidro fechado, o qual contém em seu interior um tubo de vidro pequeno no qual a água é continuamente circulada com temperatura controlada através de um banho termostatizado, propiciando o resfriamento da parte superior do tubo digestor, a fim de propiciar a recirculação dos ácidos dentro do tubo maior.

\section{Reagentes e soluções}

Todos os reagentes utilizados foram de grau analítico. A água utilizada no preparo das amostras e das soluções foi obtida por um sistema de purificação de água Direct-Q 3 (Millipore Corporation, Bedford, E.U.A.), com resistividade de $18,3 \mathrm{M} \Omega \mathrm{cm}^{-1}$. Ácido nítrico $65 \%(\mathrm{~m} / \mathrm{m})$ (Synth, Brasil) foi purificado por destilação em um sistema de sub-ebulição de quartzo MA-075 (Marconi, Piracicaba, Brasil). Antes de usar, todos os materiais de vidro foram lavados e

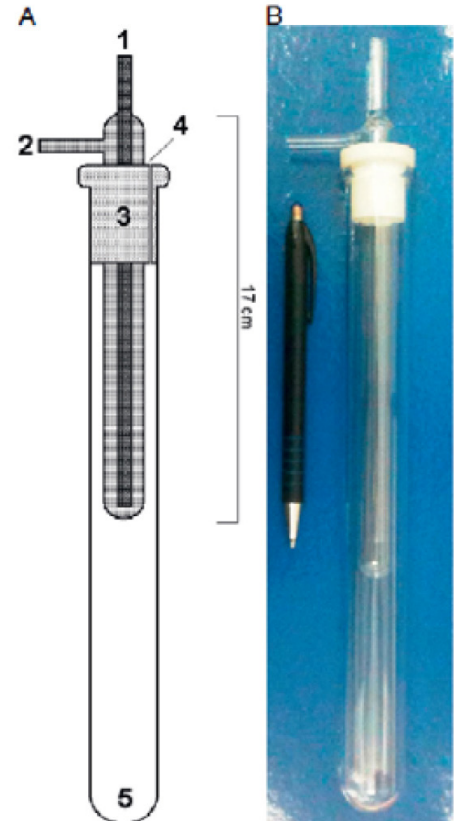

Figura 1. A) Diagrama esquemático do sistema de refluxo acoplado ao tubo digestor de vidro. 1: entrada água; 2: saída de água; 3: Tampa de Teflon ${ }^{\circledast}$; 4 : saída para gás; 5: tubo de reação. B) Imagem real do sistema de dedo frio. (adaptado de Orestes et al. 2013) ${ }^{24}$

imersos em um banho de $10 \%$ (v/v) de $\mathrm{HNO}_{3}$ durante pelo menos 48 horas e, em seguida, enxaguados com água deionizada. Foram diariamente preparadas soluções padrões de $\mathrm{Cu}$, Fe, $\mathrm{Na}$ e $\mathrm{Zn}$ a partir da diluição de uma solução estoque contendo $1000 \mathrm{mg} \mathrm{L}^{-1}$ (Fluka, Buchs, Alemanha) de todos os analitos em água deionizada a fim de realizar a calibração do instrumento.

\section{Preparo das amostras}

As amostras de carvão e cinzas utilizadas neste estudo foram cedidas pela Companhia Riograndense de Mineração (CRM), as quais foram adquiridas diretamente das minas a céu aberto de carvão mineral, que estão localizadas no município de Candiota/RS.

Para verificar a influência de cada parâmetro referente à etapa de preparo das amostras em relação ao sinal analítico de cada elemento foi realizado um planejamento estatístico utilizando o software Statistica 7.0 (StatSoft, Inc., Tulsa, OK, EUA), o qual possibilita a otimização das condições de trabalho, buscando uma condição compromisso para as variáveis em estudo: Volume de ácido nítrico (Vac), volume de peróxido de hidrogênio $(\mathrm{Vp})$, temperatura $(\mathrm{T})$ e tempo de decomposição no bloco digestor (td). Foi realizado um delineamento composto central rotacional (DCCR), $2^{4}$ com 3 pontos centrais e 8 pontos axiais, totalizando 27 ensaios realizados de forma aleatória de acordo com a ordem das análises arranjadas pelo software Statistica 7.0. As respostas selecionadas (variáveis dependentes) foram os sinais analíticos obtidos para cada elemento em estudo $(\mathrm{Cu}, \mathrm{Fe}, \mathrm{Na}$ e $\mathrm{Zn}$ ). Em todos os ensaios, a adição do volume de $\mathrm{H}_{2} \mathrm{O}_{2}(\mathrm{~mL})$ foi realizada faltando $1 / 4$ do tempo total de digestão, a fim de padronizar o procedimento.

\section{Procedimento 1: Método proposto com o sistema de refluxo}

Após a análise dos dados gerados pelos gráficos de superfície de resposta obtidos para cada analito, foi possível fixar as melhores condições de trabalho. Tendo em vista que as amostras em estudo apresentam características similares, as mesmas condições otimizadas para as amostras de carvão foram aplicadas as amostras de cinzas. 
Assim, cerca de 50,0 mg de amostras foram pesadas diretamente nos tubos digestores, a eles foram adicionados $6,0 \mathrm{~mL}$ de $\mathrm{HNO}_{3} \mathrm{e}$, posteriormente, foram levados ao aquecimento à $145^{\circ} \mathrm{C}$ em bloco digestor, com o sistema de refluxo acoplado aos tubos digestores, utilizando uma recirculação de água a $15{ }^{\circ} \mathrm{C}$. Depois de passados $45 \mathrm{~min}$, os tubos foram resfriados e adicionados $2,5 \mathrm{~mL}$ de $\mathrm{H}_{2} \mathrm{O}_{2}$, retornando ao aquecimento por mais $15 \mathrm{~min}$, totalizando um tempo de digestão final de 1 hora. A solução resultante foi transferida para frasco de PP (polipropileno) previamente calibrado e aferido a $14 \mathrm{~mL}$ com água deionizada. Todas as análises foram feitas em triplicata e, para verificar a exatidão do método proposto, testes de adição e recuperação de analitos foram realizados na presença das duas amostras em estudo.

Procedimento 2: Método recomentado pela ASTM via calcinação ${ }^{18}$

Para este procedimento, também realizado em triplicata, foi utilizado aproximadamente $1,0 \mathrm{~g}$ da amostra de carvão em cadinhos de porcelana, os quais foram calcinados por $2 \mathrm{~h}$ a $500{ }^{\circ} \mathrm{C}$ em uma mufla. Após, transferiu-se as cinzas resultantes do processo de calcinação para tubos de PTFE (Teflon) e estas foram dissolvidas na presença de $20 \mathrm{~mL}$ de água régia e $20 \mathrm{~mL} \mathrm{HF}$ concentrado, sendo a solução mantida sob aquecimento a uma temperatura de $150{ }^{\circ} \mathrm{C}$ em bloco digestor até a secura. Em seguida, adicionou-se $1,0 \mathrm{~mL} \mathrm{HNO}_{3}$ e 20 $\mathrm{mL}$ de água deionizada e levou-se ao aquecimento novamente por mais $1 \mathrm{~h}$. Ao final, a solução resultante foi transferida para o frasco de $\mathrm{PP}$ previamente calibrado e aferida à $50 \mathrm{~mL}$ com água deionizada.

\section{Estudos preliminares aplicados em amostra de carvão mineral}

Neste trabalho, primeiramente foi realizado um estudo sobre a perda de massa pela umidade característica da amostra, tendo em vista que esta é uma variável importante, pois a quantidade de energia absorvida pela amostra está diretamente relacionada ao seu teor de umidade. Em amostras desidratadas o aquecimento não é eficiente, o que pode vir a dificultar a interação com a mistura ácida durante o processo de mineralização. ${ }^{28}$

Para este estudo, cerca de $1,0 \mathrm{~g}$ de amostra de carvão mineral foi pesado em um vidro de relógio, em triplicata, e levado até uma estufa, na qual foi seco a uma temperatura de aproximadamente $105^{\circ} \mathrm{C}$ por 90 min e permaneceu em um dessecador até a pesagem à temperatura ambiente. Este processo foi realizado até as amostras adquirirem peso constante, eliminando assim a umidade, conforme é descrito pela NBR 8293 da ABNT. ${ }^{29}$ Posteriormente, foi realizado o estudo das cinzas resultantes, o qual elimina a matéria orgânica das amostras de carvão. Este procedimento foi realizado baseando-se na NBR 8289 da ABNT. ${ }^{30}$

\section{RESULTADOS E DISCUSSÕES}

\section{Umidade e teor de cinzas das amostras de carvão}

Os resultados obtidos tanto com relação ao teor de umidade quanto para o teor de cinzas na amostra de carvão analisada foram condizentes com os valores citados na literatura para esta matriz. De acordo com Pires e colaboradores, ${ }^{31}$ que realizaram um estudo de caracterização de amostras de carvão e cinzas oriundas das minas de Candiota, foram encontrados teores de aproximadamente 50\% e $16 \% \mathrm{~m} / \mathrm{m}$, para o teor de cinzas e umidade, respectivamente. Ao comparar com os valores obtidos neste trabalho, pode-se observar a semelhança nos resultados, os quais foram de aproximadamente $54 \%$ e $15 \% \mathrm{~m} / \mathrm{m}$, para as cinzas e umidade, respectivamente.

\section{Otimização do método proposto}

A otimização das melhores condições de trabalho para realizar a decomposição ácida das amostras de carvão no sistema de refluxo foi realizada através de um delineamento estatístico experimental considerando as seguintes variáveis independentes: volume de $\mathrm{HNO}_{3}$, volume de $\mathrm{H}_{2} \mathrm{O}_{2}$, tempo de decomposição e temperatura do bloco, de acordo com a Tabela 1 , cujos valores dos níveis foram determinados através de testes preliminares e também por investigação na literatura.

A matriz do DCCR (Tabela 2) contem os ensaios com as combinações das variáveis e os resultados obtidos para os quatro analitos quantificados em amostras de carvão mineral após o processo de decomposição ácida.

De acordo com a análise estatística dos dados, representada pelos Diagramas de Pareto (Figura 2), pode-se verificar que as variáveis estatisticamente significativas para o $\mathrm{Cu}$ foram: volume de ácido nítrico, tempo e temperatura. Já para o Fe, apenas o volume de ácido nítrico e o tempo foram estatisticamente significativos. Para o $\mathrm{Zn}$, as variáveis estatisticamente significativas foram as interações entre volume de ácido, tempo e temperatura. Para o Na todas as variáveis foram estatisticamente significativas. As equações 1, 2, 3 e 4 correspondem aos modelos referentes à determinação para $\mathrm{Cu}, \mathrm{Fe}, \mathrm{Na}$ e Zn.

$$
\begin{aligned}
& \mathrm{y}=0,21-0,007 \mathrm{Vac}-0,006 \mathrm{Vac}^{2}-0,004 \mathrm{td}^{2}-0,005 \mathrm{~T}^{2} \\
& \mathrm{y}=1,05-0,009 \mathrm{Vac}-0,003 \mathrm{Vp}^{2}-0,006 \mathrm{td}^{2}-0.003 \mathrm{~T}^{2} \\
& \mathrm{y}=0,21-0,007 \mathrm{Vac}-0,006 \mathrm{Vac}^{2}-0,004 \mathrm{td}^{2}-0,005 \mathrm{~T}^{2} \\
& \mathrm{y}=0,27-0,007 \mathrm{Vac}+0,007 \mathrm{~T}+0,010 \mathrm{Vac} \mathrm{td}+0,16 \mathrm{Vac} \mathrm{T}+ \\
& 0,017 \mathrm{td} \mathrm{T}
\end{aligned}
$$

Através da tabela ANOVA (Tabela 3) foi possível calcular estatisticamente alguns parâmetros importantes como, por exemplo, o valor de F. Os valores de F obtidos para todos os elementos estudados foram maiores que o valor de $\mathrm{F}$ tabelado. Com este requisito (Fcal $>$ Ftab) foi possível gerar os gráficos de superfícies de respostas e avaliar uma possível condição compromisso, levando em consideração as melhores condições de análise para cada elemento.

Desta forma, avaliando os gráficos de superfície de respostas obtidos para $\mathrm{Cu}$ e $\mathrm{Fe}$, exemplificados pelas Figuras 3 e 4, verifica-se que as melhores condições tendem aos pontos centrais. No entanto, cabe salientar que os ensaios apresentaram pouca variação entre as respostas (sinal de absorção), variando de 0,1778 a 0,2294 para o $\mathrm{Cu}$ e de 1,0167 a 1,0741 para o $\mathrm{Fe}$, indicando que qualquer condição utilizada para estes elementos resultam em uma eficiente quantificação.

Tabela 1. Variáveis e valores utilizados no DCCR

\begin{tabular}{lccccc}
\hline Variáveis independentes & \multicolumn{5}{c}{ Nível } \\
\cline { 2 - 6 } & -2 & -1 & 0 & +1 & +2 \\
\hline Volume de $\mathrm{HNO}_{3}(\mathrm{~mL})$ & 5 & 6 & 7 & 9 & 2 \\
Volume de $\mathrm{H}_{2} \mathrm{O}_{2}(\mathrm{~mL})$ & 0,5 & 1 & 1,5 & 2,5 & 120 \\
Tempo de digestão $(\mathrm{min})$ & 30 & 60 & 150 & 195 & 220 \\
Temperatura $\left({ }^{\circ} \mathrm{C}\right)$ & 120 & 145 & 170 & & 20 \\
\hline
\end{tabular}


Tabela 2. Matriz do DCCR para o processo de preparo de amostras de carvão mineral com o sistema de refluxo proposto

\begin{tabular}{|c|c|c|c|c|c|c|c|c|}
\hline \multirow{2}{*}{ Ensaios } & \multicolumn{4}{|c|}{ Variáveis Independentes } & \multicolumn{4}{|c|}{ Variáveis Dependentes } \\
\hline & Vac & $\mathrm{Vp}$ & Tempo & Temperatura & $\mathrm{Cu}^{*}$ & $\mathrm{Na}^{* * *}$ & $\mathrm{Zn*}$ & $\mathrm{Fe}^{*}$ \\
\hline 1 & $-1,0$ & $-1,0$ & $-1,0$ & $-1,0$ & 0,2030 & 1806,7 & 0,3084 & 1,0349 \\
\hline 2 & 1,0 & $-1,0$ & $-1,0$ & $-1,0$ & 0,1787 & 1969,3 & 0,2471 & 1,0167 \\
\hline 3 & $-1,0$ & 1,0 & $-1,0$ & $-1,0$ & 0,1783 & 2576,4 & 0,2927 & 1,0402 \\
\hline 4 & 1,0 & 1,0 & $-1,0$ & $-1,0$ & 0,1713 & 2591,8 & 0,2481 & 1,0300 \\
\hline 5 & $-1,0$ & $-1,0$ & 1,0 & $-1,0$ & 0,1979 & 2018,4 & 0,2789 & 1,0452 \\
\hline 6 & 1,0 & $-1,0$ & 1,0 & $-1,0$ & 0,1726 & 2077,4 & 0,2374 & 1,0293 \\
\hline 7 & $-1,0$ & 1,0 & 1,0 & $-1,0$ & 0,2017 & 2527,8 & 0,2829 & 1,0496 \\
\hline 8 & 1,0 & 1,0 & 1,0 & $-1,0$ & 0,1817 & 2640,6 & 0,2432 & 1,0418 \\
\hline 9 & $-1,0$ & $-1,0$ & $-1,0$ & 1,0 & 0,2043 & 2093,3 & 0,2840 & 1,0461 \\
\hline 10 & 1,0 & $-1,0$ & $-1,0$ & 1,0 & 0,1778 & 2065,8 & 0,2460 & 1,0403 \\
\hline 11 & $-1,0$ & 1,0 & $-1,0$ & 1,0 & 0,1840 & 2539,4 & 0,2517 & 1,0528 \\
\hline 12 & 1,0 & 1,0 & $-1,0$ & 1,0 & 0,1927 & 2541,1 & 0,2600 & 1,0257 \\
\hline 13 & $-1,0$ & $-1,0$ & 1,0 & 1,0 & 0,1897 & 2068,8 & 0,2653 & 1,0660 \\
\hline 14 & 1,0 & $-1,0$ & 1,0 & 1,0 & 0,1879 & 2043,0 & 0,3452 & 1,0221 \\
\hline 15 & $-1,0$ & 1,0 & 1,0 & 1,0 & 0,2075 & 2569,3 & 0,3135 & 1,0412 \\
\hline 16 & 1,0 & 1,0 & 1,0 & 1,0 & 0,1902 & 2549,7 & 0,3467 & 1,0273 \\
\hline 17 & $-2,0$ & 0,0 & 0,0 & 0,0 & 0,2178 & 2370,0 & 0,2958 & 1,0741 \\
\hline 18 & 2,0 & 0,0 & 0,0 & 0,0 & 0,1821 & 2204,0 & 0,2554 & 1,0259 \\
\hline 19 & 0,0 & $-2,0$ & 0,0 & 0,0 & 0,2150 & 1673,7 & 0,2834 & 1,0606 \\
\hline 20 & 0,0 & 2,0 & 0,0 & 0,0 & 0,2071 & 2851,9 & 0,2916 & 1,0239 \\
\hline 21 & 0,0 & 0,0 & $-2,0$ & 0,0 & 0,2040 & 2277,2 & 0,2864 & 1,0223 \\
\hline 22 & 0,0 & 0,0 & 2,0 & 0,0 & 0,2110 & 2332,0 & 0,2774 & 1,0400 \\
\hline 23 & 0,0 & 0,0 & 0,0 & $-2,0$ & 0,2018 & 2210,2 & 0,2362 & 1,0481 \\
\hline 24 & 0,0 & 0,0 & 0,0 & 2,0 & 0,2038 & 2202,9 & 0,2440 & 1,0366 \\
\hline 25 & 0,0 & 0,0 & 0,0 & 0,0 & 0,2290 & 2230,2 & 0,2730 & 1,0599 \\
\hline 26 & 0,0 & 0,0 & 0,0 & 0,0 & 0,2100 & 1936,9 & 0,2657 & 1,0562 \\
\hline 27 & 0,0 & 0,0 & 0,0 & 0,0 & 0,2294 & 1941,7 & 0,2804 & 1,0591 \\
\hline
\end{tabular}

*Valores referentes aos sinais de absorvância; **Valores referentes aos sinais de emissão.

Os melhores resultados para o $\mathrm{Zn}$ foram obtidos quando foram utilizados os valores das extremidades das variáveis Vac, td e T (entre os pontos -1 e $-2 ;+1$ e +2 ), conforme exemplificada na Figura 5.

Pode-se observar na Figura 6, como em todos os gráficos de superfície de resposta gerados para o $\mathrm{Na}$, que as melhores condições tendem aos pontos extremos estudados. Os melhores resultados para o $\mathrm{Na}$ foram obtidos quando utilizados os níveis $-2 \mathrm{ou}+2$ para o Vac, td e T. Para o Vp a melhor condição foi no nível +2 . Entretanto, pode-se observar que não houve grande variação no sinal de emissão obtido nos ensaios. Portanto, as condições para validação foram baseadas nos resultados dos outros elementos.

Após a avaliação dos gráficos de superfície gerados para os elementos em estudo, pode-se buscar uma condição compromisso para uma tentativa de determinação dos quatro analitos em um único preparo de amostras. Assim, foi fixado um volume maior de $\mathrm{H}_{2} \mathrm{O}_{2}(2,5$ $\mathrm{mL}$ ), visto que foi uma condição fundamental para a determinação de $\mathrm{Na}$ e para os demais foi verificado que não alteraria significativamente na determinação dos mesmos. As outras condições (temperatura, tempo e volume de $\mathrm{HNO}_{3}$ ) foram estabelecidas levando em consideração os resultados para a determinação de $\mathrm{Zn}$, as quais foram fixadas em $6,0 \mathrm{~mL} \mathrm{HNO}_{3}$, a uma temperatura de $145^{\circ} \mathrm{C}$ por $60 \mathrm{~min}$. É importante salientar que, mesmo não sendo as condições ótimas observadas para os demais elementos, como $\mathrm{Cu}$ e $\mathrm{Fe}$, que apontavam para os pontos centrais, estas condições ainda demonstraram estar dentro de uma faixa de boa resposta, permitindo assim o uso destas condições de trabalho.

De acordo com a literatura, de maneira geral, o carvão e as cinzas apresentam normalmente os metais na forma de sulfetos, podendo apresentar também carbonatos, sulfatos, entre outros, os quais são facilmente extraídos em condições ácidas. Em função disso, a temperatura de $145^{\circ} \mathrm{C}$ por 60 minutos foi suficiente para romper a ligação dos metais com os grupamentos e liberá-los em solução, os quais foram certificados pelos testes de recuperação. $\mathrm{O}$ volume de $2,5 \mathrm{~mL}$ de $\mathrm{H}_{2} \mathrm{O}_{2}$ foi necessário devido a amostra possuir alta carga orgânica, auxiliando o processo de decomposição iniciado pelo $\mathrm{HNO}_{3}$.

O uso do DCCR como ferramenta estatística possibilitou a determinação da condição otimizada do processo de decomposição. A análise dos dados permitiu a seleção de uma condição com um menor consumo de reagentes, menor tempo, bem como uma boa qualidade nos resultados. Além disso, foi possível realizar um menor número de ensaios quando comparado ao método de tentativa e erro ou a combinação de todos os fatores. Cabe salientar que todas as respostas foram avaliadas simultaneamente, o que possibilitou a determinação da mesma condição para todos os analitos estudados.

\section{Parâmetros de mérito}

Os parâmetros de mérito obtidos para a determinação de $\mathrm{Cu}$, $\mathrm{Fe}, \mathrm{Na}$ e $\mathrm{Zn}$ em amostras de carvão e cinzas por F AAS a partir da metodologia proposta estão apresentados na Tabela 4. Como pode ser observado, as curvas de calibração para todos os analitos estudados apresentaram bons coeficientes de correlação linear, com R > 0,99, bem como mostraram LDs, LQs e sensibilidades adequadas para atender ao objetivo que o trabalho se propõe. 
$\mathrm{Cu}$
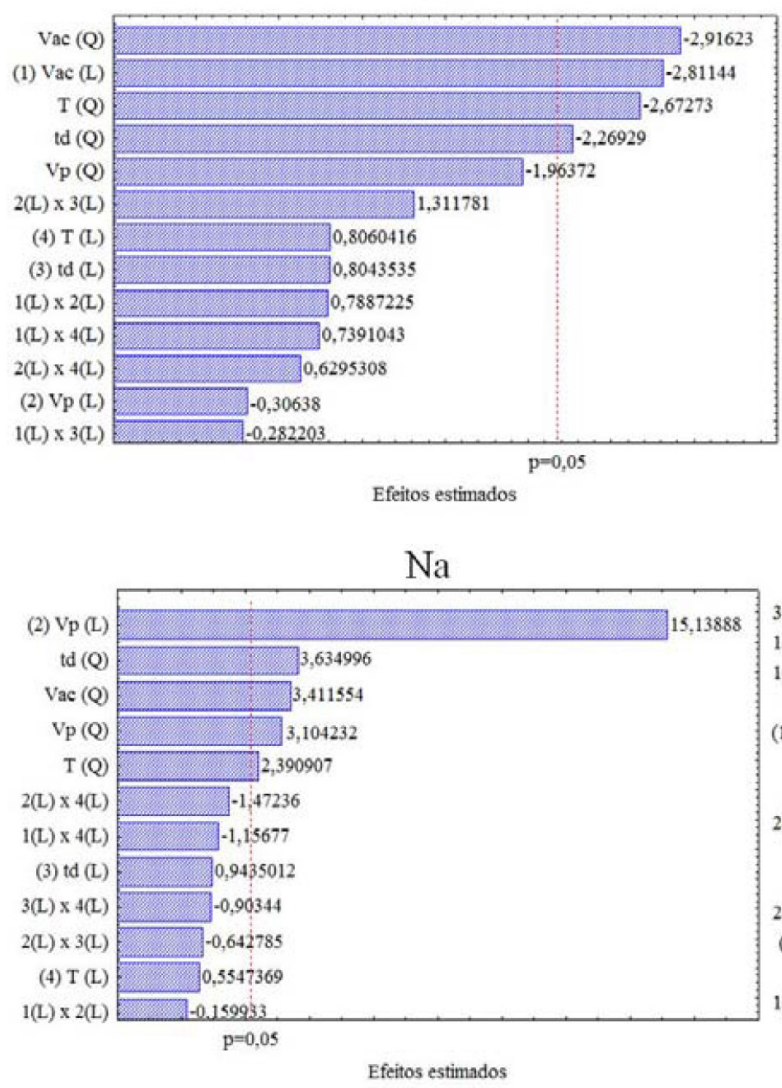

$\mathrm{Fe}$

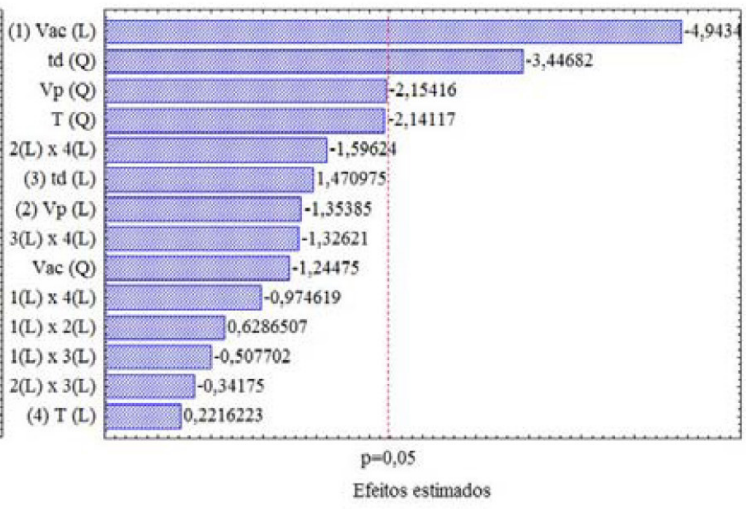

$\mathrm{Zn}$

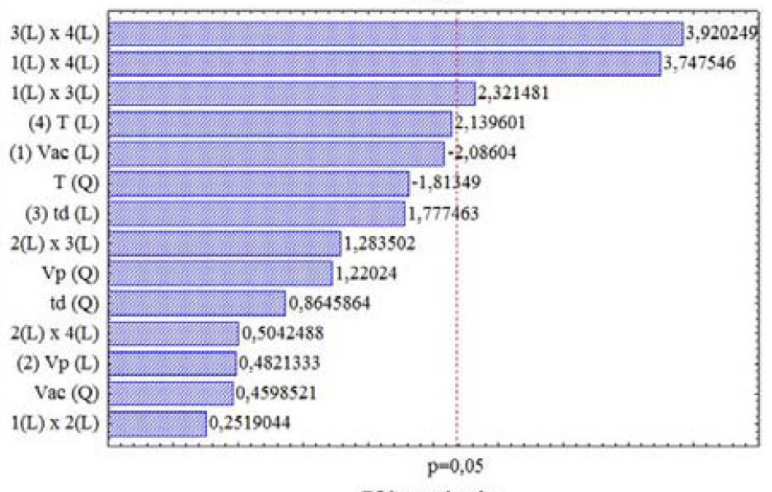

Figura 2. Gráficos de Pareto para $\mathrm{Cu}, \mathrm{Fe}$, Na e Zn

Tabela 3. Análise de variância (ANOVA) para a determinação de $\mathrm{Cu}, \mathrm{Fe}, \mathrm{Na}$ e Zn em amostra de carvão mineral

\begin{tabular}{|c|c|c|c|c|c|}
\hline Respostas & Fonte de variação & Soma dos quadrados & Graus de liberdade & Quadrado médio & Fcal \\
\hline \multirow{3}{*}{$\mathrm{Cu}$} & Regressão & 0,00305 & 4 & 0,00076 & \multirow{3}{*}{4,75} \\
\hline & Resíduos & 0,00372 & 22 & 0,00016 & \\
\hline & Total & 0,00677 & 26 & & \\
\hline \multirow{3}{*}{$\mathrm{Fe}$} & Regressão & 0,00357 & 4 & 0,00089 & \multirow{3}{*}{8,55} \\
\hline & Resíduos & 0,00230 & 22 & 0,00010 & \\
\hline & Total & 0,00588 & 26 & & \\
\hline \multirow{3}{*}{$\mathrm{Na}$} & Regressão & 207211 & 5 & 414423 & \multirow{3}{*}{58,4} \\
\hline & Resíduos & 148911 & 21 & 7091 & \\
\hline & Total & 222102 & 26 & & \\
\hline \multirow{3}{*}{$\mathrm{Zn}$} & Regressão & 0,0143 & 5 & 0,00286 & \multirow{3}{*}{7,24} \\
\hline & Resíduos & 0,0083 & 21 & 0,00039 & \\
\hline & Total & 0,0226 & 26 & & \\
\hline
\end{tabular}

$\mathrm{F}_{4,22}=2,82 ; \mathrm{F}_{5,21}=2,68$.

\section{Aplicação das condições otimizadas e validação da metodologia proposta}

Com o objetivo de avaliar a exatidão da metodologia proposta do preparo das amostras estudadas foram utilizadas amostras de carvão e cinzas fortificadas e não fortificadas. A Tabela 5 apresenta os resultados dos testes de adição e recuperação para $\mathrm{Cu}, \mathrm{Fe}, \mathrm{Na}$ e $\mathrm{Zn}$ em ambas as amostras, bem como as concentrações encontradas para estes elementos nas amostras em estudo.

Tendo como base os percentuais obtidos nos testes de adição e recuperação para o carvão, apresentados na Tabela 5 , os quais variam entre 92,7 a $104,7 \%$, foi possível verificar que a metodologia proposta apresenta exatidão para análise. Além disso, foram encontrados baixos valores de desvio padrão relativos (RSD), sendo estes inferiores a $6 \%$, o que evidencia uma boa precisão dos resultados.

Conforme citado anteriormente, as mesmas condições otimizadas para decomposição das amostras de carvão também foram aplicadas para análise de cinzas, apresentando exatidão e precisão adequada para determinação dos respectivos analitos, o que pode ser visto a partir dos resultados apresentados na Tabela 5, na qual os percentuais de recuperação variaram entre 98,7 e 115,3\% e RSDs menores que 4\%.

Através da análise dos resultados apresentados na Tabela 5 também pode-se verificar que a amostra de cinzas em estudo encontra-se dentro dos limites máximos permitidos para $\mathrm{Cu}, \mathrm{Zn}$ e $\mathrm{Na}$ de acordo com a normativa vigente, ${ }^{7}$ a qual classifica esta matriz em resíduos sólidos classe II A - Não inerte. Porém, o valor obtido para o Fe 


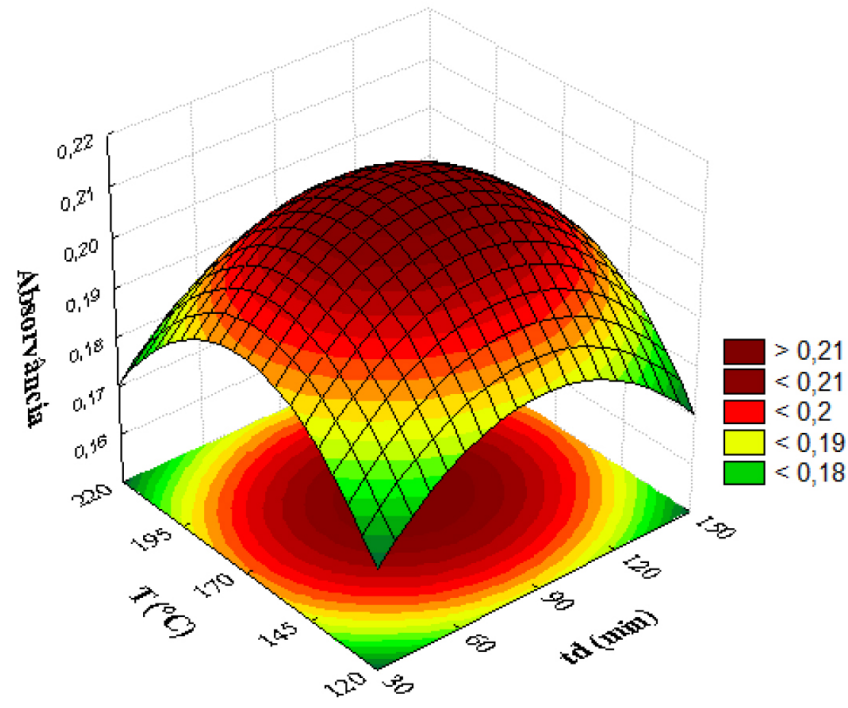

Figura 3. Gráfico de superfície de resposta em função do sinal analítico do $\mathrm{Cu}(\mathrm{Txt})$

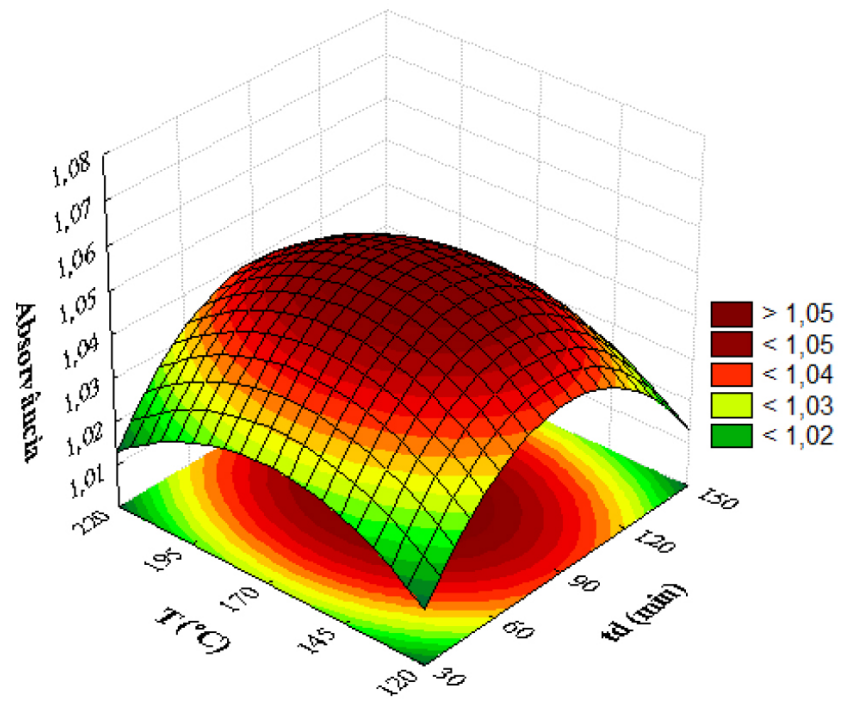

Figura 4. Gráfico de superfície de resposta em função do sinal analítico do $\mathrm{Fe}(\mathrm{Txt})$

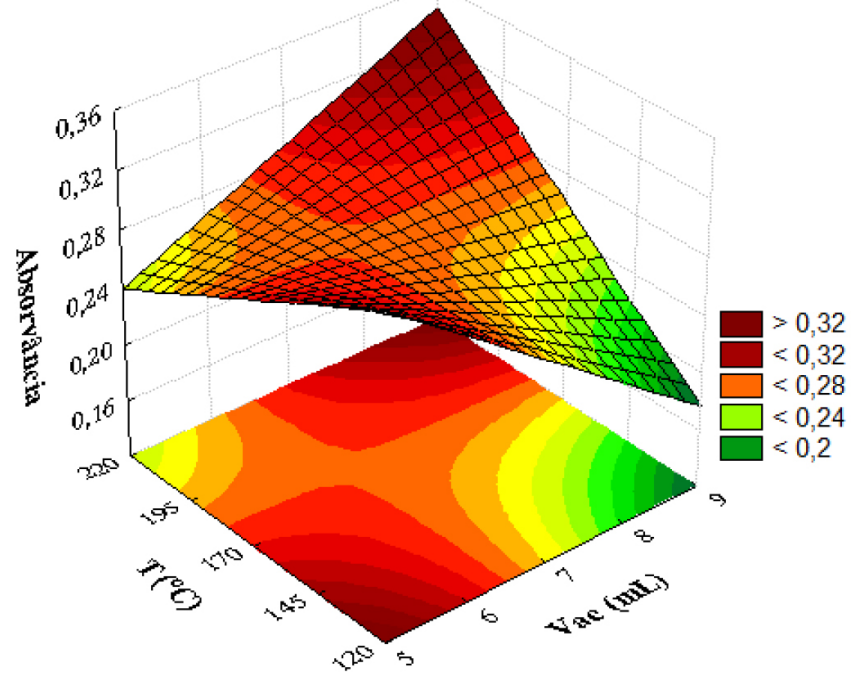

Figura 5. Gráfico de superfície de resposta em função do sinal analítico do $\mathrm{Zn}(\mathrm{Tx} V a c)$

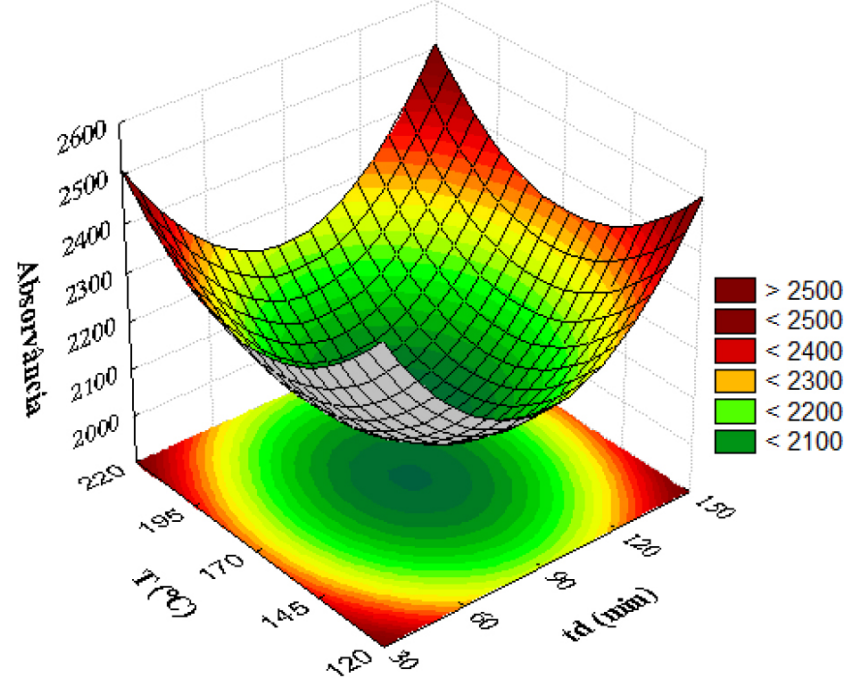

Figura 6. Gráfico de superfície de resposta em função do sinal analítico do $\mathrm{Na}(\mathrm{Txt} d)$

Tabela 4. Parâmetros de Mérito para $\mathrm{Cu}, \mathrm{Fe}, \mathrm{Zn}$ e Na por técnicas espectrométricas em carvão e cinzas

\begin{tabular}{lccccc}
\hline Analito & Faixa Linear $\left(\mathrm{mg} \mathrm{L}^{-1}\right)$ & $\mathrm{a}\left(\mathrm{L}^{-1} \mathrm{mg}\right)$ & $\mathrm{LD}\left(\mathrm{mg} \mathrm{L}^{-1}\right)$ & $\mathrm{LQ}\left(\mathrm{mg} \mathrm{L}^{-1}\right)$ & 0,079 \\
$\mathrm{Cu}$ & $0,5-3,0$ & 0,056 & 0,023 & 0,128 & 0,999 \\
$\mathrm{Fe}$ & $1,0-5,0$ & 0,032 & 0,038 & 0,010 & 0,999 \\
$\mathrm{Zn}$ & $0,25-1,0$ & 0,218 & 0,003 & 0,097 & 0,998 \\
$\mathrm{Na}$ & $1,0-5,0$ & 389,0 & 0,029 & 0,998 \\
\hline
\end{tabular}

a: coeficiente angular da curva; LD: limite de detecção; LQ: limite de quantificação; R: coeficiente da curva de calibração.

(23,79 $\left.\mathrm{mg} \mathrm{L}^{-1}\right)$ encontra-se bem acima do valor estipulado pela normativa, sendo que o máximo permitido é de $0,3 \mathrm{mg} \mathrm{L}^{-1}$, salientando a importância de controlar a concentração de metais nestas amostras, a fim de prevenir maiores problemas ambientais pelo descarte indevido destes resíduos.

Além disso, foi aplicada uma metodologia oficial recomendada pela ASTM para análise de carvão mineral, a fim de comprovar a exatidão do método proposto, através da comparação das duas metodologias. Na Tabela 6 , pode-se observar que os valores obtidos através dos dois métodos de preparo das amostras estão de acordo para os analitos em estudo, indicando a exatidão dos resultados, uma vez que não houve diferenças significativas entre os resultados a um nível de confiança de $95 \%$, atestado pela aplicação do teste $t$-student pareado, com valores de t calculados $(1,98 ; 1,62 ; 1,56$ e 0,33 para $\mathrm{Cu}, \mathrm{Fe}, \mathrm{Na}$ e $\mathrm{Zn}$, respectivamente $)<\mathrm{t}$ crítico $(4,30)$.

Neste trabalho foi possível verificar a eficiência do uso da decomposição ácida com sistema de refluxo nas amostras de carvão e cinzas. Essa eficiência no processo pode ser atribuída à zona de 
Tabela 5. Concentrações obtidas em mg L ${ }^{-1}$ de $\mathrm{Cu}, \mathrm{Fe}, \mathrm{Zn}$ e $\mathrm{Na}$ em carvão e cinzas, média \pm desvio (RSD) e \% de recuperação

\begin{tabular}{|c|c|c|c|c|}
\hline & média \pm desvio $(\mathrm{RSD})$ & Recuperação (\%) & média \pm desvio (RSD) & Recuperação (\%) \\
\hline & \multicolumn{2}{|c|}{ Amostras de carvão } & \multicolumn{2}{|c|}{ Amostras de cinzas } \\
\hline \multicolumn{5}{|c|}{$\mathbf{C u}$} \\
\hline Sem adição & $0,092 \pm 0,001(1,1)$ & 104,7 & $0,148 \pm 0,004(2,7)$ & 115,3 \\
\hline Adição de 1,5 ppm & $1,663 \pm 0,026(1,6)$ & & $1,877 \pm 0,022(1,2)$ & \\
\hline \multicolumn{5}{|c|}{$\mathrm{Fe}$} \\
\hline Sem adição & $37,11 \pm 0,76(2,1)$ & 93,6 & $23,79 \pm 0,86(3,6)$ & 113,7 \\
\hline Adição de 3,0 ppm & $39,92 \pm 0,39(0,9)$ & & $27,20 \pm 0,39(1,4)$ & \\
\hline \multicolumn{5}{|c|}{$\mathbf{Z n}$} \\
\hline Sem adição & $0,288 \pm 0,002(0,7)$ & 96,0 & $0,128 \pm 0,004(3,1)$ & 114,8 \\
\hline Adição de 0,5 ppm & $0,768 \pm 0,026(3,4)$ & & $0,702 \pm 0,012(1,7)$ & \\
\hline \multicolumn{5}{|c|}{$\mathrm{Na}$} \\
\hline Sem adição & $0,696 \pm 0,013(1,9)$ & 92,7 & $5,930 \pm 0,085(1,4)$ & 98,7 \\
\hline Adição de 3,0 ppm & $3,477 \pm 0,182(5,2)$ & & $8,890 \pm 0,028(0,3)$ & \\
\hline
\end{tabular}

Tabela 6. Comparação dos resultados obtidos de $\mathrm{Cu}, \mathrm{Fe}, \mathrm{Na}$ e Zn em carvão por F AAS entre o método proposto e o método oficial recomendado

\begin{tabular}{lcccc}
\hline & \multicolumn{2}{c}{ Método Proposto (Dedo Frio) } & \multicolumn{2}{c}{ Método Oficial (ASTM) } \\
\cline { 2 - 5 } & Concentração $\left(\mathrm{mg} \mathrm{kg}^{-1}\right)$ & RSD $(\%)$ & Concentração $\left(\mathrm{mg} \mathrm{kg}^{-1}\right)$ & RSD $(\%)$ \\
\hline $\mathrm{Cu}$ & $25,9 \pm 0,4$ & 1,5 & $24,1 \pm 1,9$ & 7,9 \\
$\mathrm{Fe}$ & $10487,4 \pm 184,0$ & 1,8 & $11770,5 \pm 66,0$ & 0,6 \\
$\mathrm{Na}$ & $203,3 \pm 8,3$ & 4,1 & $229,0 \pm 14,8$ & 6,5 \\
$\mathrm{Zn}$ & $80,7 \pm 0,4$ & 0,5 & $78,9 \pm 9,6$ & 12,2 \\
\hline
\end{tabular}

$\mathrm{n}=3$, para ambos métodos.

baixa temperatura no dedo frio, proporcionando a condensação dos vapores gerados, aumentando a capacidade de decomposição sem a necessidade de reposição dos ácidos. Provavelmente, na superfície do dedo frio, é gerada uma película fina de nitratos (pela decomposição com o ácido nítrico), permitindo a absorção dos analitos. Além disso, o sistema também apresenta uma ranhura para alivio de pressão, diminuindo ou anulando qualquer risco de explosão, tornando-se um método mais seguro ao analista. O método, quando comparado à metodologia oficial, reduz em $80 \%$ o tempo de análise e $70 \%$ do consumo de reagentes, permitindo que sejam avaliados elementos sem o risco de contaminação e perdas.

\section{CONCLUSÃO}

A metodologia apresentada para a decomposição ácida de amostras de carvão e cinzas, baseada no uso de um sistema de refluxo para determinação de $\mathrm{Cu}, \mathrm{Fe}, \mathrm{Na}$ e $\mathrm{Zn}$ por técnicas de Espectrometria Atômica (F AAS e F AES), demonstrou ser um método simples, eficiente, rápido e seguro, utilizando poucas quantidades de amostras e reagentes quando comparada a outras metodologias convencionais já utilizadas. O uso do planejamento estatístico possibilitou a otimização do processo de decomposição das amostras com um numero de ensaios menor do que com outras metodologias de avaliação das variáveis, além de maior informação com relação aos fatores estudados. A eficiência do método proposto também pôde ser avaliada, apresentando boas taxas de recuperação dos analitos em estudo, tanto para amostras de carvão quanto para amostras de cinzas, atestando uma boa exatidão da metodologia. Além disso, a exatidão também foi verificada através da comparação dos resultados com a metodologia oficial recomendada para carvão.

\section{AGRADECIMENTOS}

Os autores agradecem ao financiamento proporcionado pelo
Projeto Casadinho $\mathrm{n}^{\circ}$. 552197/2011-4) e pelas bolsas concedidas pelos órgãos de fomento CNPq (Conselho Nacional de Pesquisa e Desenvolvimento Tecnológico) e a CAPES (Coordenação de Aperfeiçoamento de Pessoal de Nível Superior).

\section{REFERÊNCIAS}

1. http://www.carvaomineral.com.br/arquivos/assembleia_RS.pdf, acessada em Janeiro/2015.

2. Baird, C.; Química Ambiental, 2a ed., Bookman: Porto Alegre, 2002.

3. Sager, M.; Toxicol. Environ. Chem. 1999, 71, 159.

4. Finkelman, R. B.; Gross, P. M. K.; Int. J. Coal Geol. 1999, 40, 91.

5. Swaine, D. J.; Goodarzi, F.; Environmental aspects of trace elements in coal, Kluwer Academia Publishers: Dordrecht, 1995.

6. Valkovic, V.; Trace elements in coal, CRC Press: Boca Raton, 1983.

7. ABNT (Associação Brasileira de Normas Técnicas) - NBR 10004 Resíduos Sólidos: Classificação; Rio de Janeiro, 2004.

8. Krug, F. J.; Em Métodos de Preparo de Amostras: Fundamentos sobre preparo de amostras orgânicas e inorgânicas para análise elementar, $1^{\text {a }}$ ed., Piracicaba: Edição do autor, São Paulo, 2010, cap. 5.

9. Oliveira, E.; J. Braz. Chem. Soc. 2003, 14, 174.

10. Mujuru, M.; Moyo, S.; McCrindle, R. I.; Mokgalaka-Matlala, N.; Spectrochim. Acta, Part B 2012, 76, 147.

11. Meeravali, N. N.; Madhavi, K.; Kumar, S. J.; Talanta 2013, 104, 180.

12. Maia, S.M.; Pozebon, D.; Curtius, A. J.; J. Anal. At. Spectrom. 2003, 18, 330.

13. Borges, D. L. G.; Welz, B.; Curtius, A. J.; Microchim. Acta 2007, 159, 19.

14. Martins. P.; Pozebon. D.; Dressler, V. L.; Kemieciki G. A.; Anal. Chim. Acta 2002, 470, 195.

15. Ghisi, M.; Ribeiro, A. S.; Vieira, M. A.; Curtius, A. J.; Revista Analytica 2007, 28, 58.

16. Damin, I. C. F.; Silva, M. M.; Vale, M. G. R.; Welz, B.; Spectrochim. Acta, Part B 2007, 62, 1037. 
17. Mior, R.; Morés, S.; Welz, B.; Carasek, E.; Andrade, J. B.; Talanta 2013, 104, 368.

18. Annual Book of ASTM Standards, D 6357-00a, 2000.

19. Annual Book of ASTM Standards, D 6349-01, 2001.

20. Annual Book of ASTM Standards, D 3683-94, 2000.

21. Bettinelli, M.; Baroni, U.; Pastorelli, N.J.; Anal. At. Spectrom. 1988, 3, 1005.

22. Wang, J.; Nakazato, T.; Kinya, S.; Yamada, O.; Tao, H.; Saito, I.; Anal. Chim. Acta 2004, 514, 115.

23. Antes, F. G.; Duarte, F. A.; Mesko, M. F.; Nunes, M. A. G.; Pereira, V. A.; Müller, E. I.; Dressler, V. L.; Flores, E. M. M.; Talanta 2010, 83, 364.

24. Oreste, E. Q.; Jesus, A.; Oliveira, R. M.; Silva, M. M.; Vieira, M. A.; Ribeiro, A. S.; Microchem. J. 2013, 109, 5.

25. Oreste, E. Q.; Souza, A. O.; Pereira, C. C.; Lisboa, M. T.; Cidade, M. J. A.; Vieira, M. A.; Cadore. S.; Ribeiro, A. S.; Food Anal. Methods 2015, 9, 777 .
26. Oliveira, R. de M.; Antunes, A. C. N.; Vieira, M. A.; Medina, A. L.; Ribeiro, A. S.; Microchem. J. 2016, 124, 402.

27. Box, G. E. P.; Hunter, W. G.; Hunter, J. S.; Statistics for experimenters: An introduction to designs, data analysis and model building, Wiley: New York, 1978.

28. Casada, M. E.; Walton, L. R.; Swetnam, L. D.; Casada, J. H.; Trans. ASAE 1983, 907.

29. ABNT (Associação Brasileira de Normas Técnicas) - Carvão Mineral: Determinação de umidade. NBR 8293 (MB1893), 1983.

30. ABNT (Associação Brasileira de Normas Técnicas) - Carvão Mineral: Determinação do teor de cinzas. NBR 8289 (MB1891), 1983.

31. Pires, M.; Querol, X.; Teixeira, E. C.; Geochim. Bras. 2001, 15, 113. 